\title{
Shufeng Jiedu Capsules Alleviate Lipopolysaccharide-Induced Acute Lung Inflammatory Injury via Activation of GPR18 by Verbenalin
}

\author{
Ying Yuan ${ }^{a}$ Qingwu Liao ${ }^{b}$ Mingming Xue ${ }^{c}$ Yujing Shid ${ }^{\text {Ling Ronge }}$ \\ Zhenju Song $^{c}$ Zhaoyang Tong ${ }^{c}$ Wuhong Zheng ${ }^{f}$ Qiang Zhu ${ }^{g}$ \\ Xiaolan Cuid Zhengang Tao ${ }^{c}$
}

aDepartment of Presbyatrics, Affiliated Zhongshan Hospital, Fudan University, Shanghai, bepartment of Anesthesiology, Affiliated Zhongshan Hospital, Fudan University, Shanghai, 'Department of Emergency, Affiliated Zhongshan Hospital, Fudan University, Shanghai, dInstitute of Chinese Materia Medica, China Academy of Chinese Medical Sciences, Beijing, eDepartment of Respiratory Medicine, Bozhou People's Hospital, Bozhou, 'Department of Emergency, Fujian Provincial Hospital, Provincial Clinical Medical College of Fujian Medical University, Fuzhou, '9Anhui Jiren Pharmaceutical Co., Ltd., Bozhou, China

\section{Key Words}

Acute respiratory tract infection - GPR18 - Shufeng Jiedu Capsule • Verbenalin • Peritoneal macrophages

\footnotetext{
Abstract

Background/Aims: Acute respiratory tract infection (ARTI) is the most common reason for outpatient physician office visits. Although powerful and significant in the treatment of infections, antibiotics used for ARTI inappropriately have been an important contributor to antibiotic resistance. We previously reported that Shufeng Jiedu Capsule (SJC) can effectively amplify anti-inflammatory signaling during infection. In this study, we aimed to systematically explore its composition and the mechanism of its effects in ARTI. Methods: Pseudomonas aeruginosa (PAK) strain was used to generate a mouse model of ARTI, which were then treated with different drugs or compounds to determine the corresponding anti-inflammatory roles. High-performance liquid chromatography-quadrupole time of flight-tandem mass spectrometry. was conducted to detect the chemical compounds in SJC. RNAs from the lung tissues of mice were prepared for microarray analysis to reveal globally altered genes and the pathways involved after SJC treatment. Results: SJC significantly inhibited the expression and secretion of inflammatory factors from PAK-induced mouse lung tissues or lipopolysaccharideinduced peritoneal macrophages. Verbenalin, one of the bioactive compounds identified

Y. Yuan, Q. Liao, M. Xue, Y. Shi and L. Rong contributd equally to this work.

Dr. Zhengang Tao

and Dr. Xiaolan Cui

Department of Emergency, Affiliated Zhongshan Hospital, Fudan University

Shanghai 200032 (China); Tel. +86-17718556916,

E-Mail tao.zhengang@zs-hospital.sh.cn; Thanatoswh@163.com; cuixiaolan2812@126.com
} 


\section{Cellular Physiology Cell Physiol Biochem 2018;50:629-639 \begin{tabular}{ll|l} 
DOI: 10.1159/000494184 & O 2018 The Author(s). Published by S. Karger AG, Basel \\
www.karger.com/cpb
\end{tabular} \\ Yuan et al.: Verbenalin Inhibits Lung Inflammation by GPR18}

in SJC, also showed notable anti-inflammatory effects. Microarray data revealed numerous differentially expressed genes among the different treatment groups; here, we focused on studying the role of GPR18. We found that the anti-inflammatory role of verbenalin was attenuated in GPR18 knockout mice compared with wild-type mice, although no statistically significant difference was observed in the untreated PAK-induced mice types. Conclusion: Our data not only showed the chemical composition of SJC, but also demonstrated that verbenalin was a significant anti-inflammatory compound, which may function through GPR18.

(C) 2018 The Author(s)

Published by S. Karger AG, Basel

\section{Introduction}

Acute respiratory tract infection (ARTI) refers to an acute infectious disease involving the respiratory tract, such as acute uncomplicated pharyngitis, rhinosinusitis, bronchitis, pneumonia, and the common cold. ARTI can be classified as an acute upper respiratory tract infection (AURTI) or an acute lower respiratory tract infection (ALRTI). ALRTI is generally more serious than an AURTI and is the most common reason for outpatient physician office visits. Moreover, ALRTIs are the leading cause of death among all infectious diseases [1-3]. ALRTIs are generally treated with antibiotics, and approximately $41 \%$ of all adult ambulatory antibiotics prescriptions are for respiratory infections [4, 5]. Although antibiotics are powerful and important in the treatment of infections, inappropriate use is an important contributor to antibiotic resistance. Antibiotic misuse not only increases health care costs, but also poses a significant threat to public health.

Traditional Chinese medicines have been shown to be advantageous for the treatment of ALRTI-for example, the Shufeng Jiedu Capsule (SJC), which is made from eight traditional Chinese medicinal materials including Polygonum cuspidatum, Fructus forsythia, Radix isatidis, Radix bupleuri, Dahurian patrinia herb, Verbena, Rhizoma phragmitis and Liquorice root. SJC has been reported to have both antiviral and antibacterial effects in clinical trials [6]. Moreover, its functions in animal models have also been confirmed [7]. However, the detailed functional mechanisms for its anti-infection effects have not been elucidated. In addition, the chemical components of SJC need to be identified to improve combined treatment with traditional Chinese medicine and Western medicine.

In this study, we tested the effects of SJC on a mouse model of ALRTI and found that SJC functioned by decreasing the inflammatory factors in lung tissues. We next verified the anti-inflammatory roles of SJC by isolating mouse peritoneal macrophages and performing cellular experiments. Importantly, we initially explored the global effects of SJC by a microarray approach and found that G protein-coupled receptor 18 (GPR18) may participate in the signaling pathways. Furthermore, we identified the chemical contents of SJC by highperformance liquid chromatography-quadrupole time of flight-tandem mass spectrometry (HPLC-Q-TOF-MS/MS), and revealed that verbenalin is a potential anti-inflammatory component. Finally, we confirmed that verbenalin can function through GPR18 to inhibit inflammation by using GPR18-knockout (KO) mice.

\section{Materials and Methods}

\section{Animals and materials}

SJC, which was approved and patented as a clinical drug by the Chinese Food and Drug Administration in 2009, was purchased from Jiren Pharmaceutical (Cat. Z20090047; Anhui, China). Details on the composition of the individual components have been revealed recently [8]. We also performed an additional mass spectrometry assay to verify the components (Supplementary Table 1), which were consistent with previous data and showed that the components were uniform from batch to batch (for all supplementary material see www.karger.com/10.1159/000494184/. Enzyme-linked immunosorbent assay (ELISA) kits for interleukin-6 (IL-6) and IL-8 were purchased from UnivBio (Shanghai, China). ELISA kits for tumor necrosis factor $\alpha$ (TNF- $\alpha$ ) were purchased from Shanghai United Cell Biotechnology Corporation (Shanghai, China). 


\section{Cellular Physiology Cell Physiol Biochem 2018;50:629-639

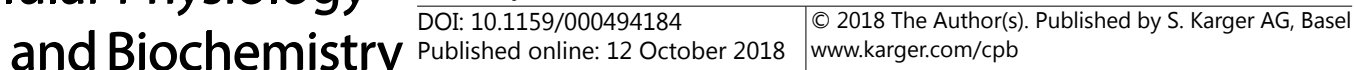

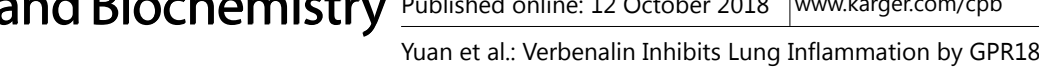

Female wild-type (WT) C57BL/6 mice aged approximately 8 weeks were purchased from Fudan University's Animal Research Center (Shanghai, China). GPR18-KO mice were purchased from Taconic Biosciences (Hudson, NY). Mice were fed normal mouse chow and water ad libitum and were maintained under standard conditions with air filtration. All animal studies were approved by the Ethics Committee of Fudan University.

\section{Bacterial culturing}

Pseudomonas aeruginosa WT PAK strain is known to induce acute lung inflammation due to its enriched inflammatory factors such as pili, flagella, exotoxin A, elastases, and phospholipases [9]. Briefly, the cells were grown in Luria-Bertani (LB) broth for $16 \mathrm{~h}$, then transferred into fresh LB medium for another $5 \mathrm{~h}$ at $37^{\circ} \mathrm{C}$. PAK cells were collected by centrifuging at 4, $000 \times g$ for $15 \mathrm{~min}$ followed by washing twice with phosphate-buffered saline (PBS). The cell pellets were diluted to the appropriate density by PBS and verified under a microscope.

\section{Mouse infections and treatments}

WT C57BL/6 mice ( $\mathrm{n}=90)$ were randomly divided into six groups: control (Con, without bacteria), model (Mod, introduction of bacteria but treatment with normal saline only), levofloxacin group (Lev), SJC high-dose group (SJC-H), SJC middle-dose group (SJC-M), and SJC low-dose group (SJC-L). All mice were anesthetized by intramuscular administration of a mixture of ketamine and xylazine. Mice in the control group were treated with intranasal mock control, while those in the other five groups were infected intranasally with bacterial doses equivalent to $5 \times 10^{6}$ colony-forming units per mouse (approximately onetenth of the $\mathrm{LD}_{50}$ ), as described previously [10].

Mice in the Lev group received intragastric administration of levofloxacin (75 mg/kg); the SJC-L, SJC-M, and SJC-H groups received intragastric SJC $(0.025 \mathrm{~g} / \mathrm{mL}, 0.05 \mathrm{~g} / \mathrm{mL}$, and $0.1 \mathrm{~g} / \mathrm{mL}$, respectively); and the Con and Mod groups were treated with normal saline. All drug treatments were administered once per day for 2 weeks. Finally, all mice were anesthetized and sacrificed.

\section{Lung tissue extraction and enzyme-linked immunosorbent assay (ELISA)}

After the mice were sacrificed, the lungs were exposed and the pulmonary arteries were perfused with PBS. The right lung was ligated at the right hilum, resected, rinsed in PBS, and lysed using RIPA buffer [11]. The levels of IL-6 and IL-8 were measured using ELISA kits according to the manufacturers' instructions.

\section{Isolation and culture of mice peritoneal macrophages}

The procedure was a slight modification of previous procedures [12, 13]. Briefly, 8-10-week-old mice received intraperitoneal injections with $1 \mathrm{~mL}$ of $4 \%$ Brewer thioglycollate medium (Difco, Detroit, MI) to increase the number of peritoneal macrophages. After 3 days of injections, peritoneal cells were isolated by washing the peritoneal cavity with $10 \mathrm{~mL}$ ice-cold Hank's balanced salt solution (HBSS) without calcium or magnesium. The cells were centrifuged at 12, $000 \mathrm{rpm}$ for $5 \mathrm{~min}$ and washed with red blood cell lysis buffer (BioLegend, San Diego, CA), followed by washing with HBSS. Cells were then suspended and seeded on 6-well cell culture dishes in medium with $10 \%$ fetal bovine serum (FBS), 1\% penicillin-streptomycin and $0.1 \%$ amphotericin $\mathrm{B}$ at $37{ }^{\circ} \mathrm{C}$ in an incubator with a humidified atmosphere of $5 \% \mathrm{CO}_{2}$. The cells were allowed to adhere for $2 \mathrm{~h}$; floating cells were washed away. After $24 \mathrm{~h}$ of culture, adherent cells were used for the indicated experiments.

\section{Microarray analysis}

RNA was extracted from the left lung tissues using Qiagen RNeasy® Lipid Tissue Mini Kits (Qiagen Sciences, Chatsworth, CA). RNA concentrations were determined by a NanoDrop spectrophotometer. Equal amounts of total RNA from each sample were labeled using an Agilent Low RNA Input Linear Amplification kit (Agilent Technologies, Santa Clara, CA) and hybridized to the Agilent oligonucleotide probe sequence, followed by reverse transcription into cDNA with a T7 promoter primer and cyanine (Cy)3-CTP labeling. Labeled cDNA was further purified using RNeasy mini purification columns (Qiagen Sciences) to remove unlabeled products. Purified cDNA from each group was fragmented for the microarray hybridizations onto Agilent Mouse Gene Expression arrays (8×60K, Design ID: 028005). The arrays were scanned on an Agilent scanner and processed using Feature Extraction and GeneSpring software. 


\section{Cellular Physiology Cell Physiol Biochem 2018;50:629-639 \begin{tabular}{l|l|l} 
DOI: 10.1159/000494184 & $\begin{array}{l}\text { O 2018 The Author(s). Published by S. Karger AG, Basel } \\
\text { www.karger.com/cpb }\end{array}$
\end{tabular} \\ Yuan et al.: Verbenalin Inhibits Lung Inflammation by GPR18}

Ingenuity pathway analysis

An integrated analysis using multiple datasets was performed to assemble SJC-induced gene regulatory network in the lungs. Genes with $>2$-fold or $<0.5$-fold changes were first selected and enriched using Gene Ontology and KEGG database to generate a heat map. To clarify further molecular connections between the proteins, protein-protein interaction (PPI) data were then extracted from the String database (http:// string-db.org) using an in-house Python script.

\section{Time of flight-mass spectrometry}

An HPLC-Q-TOF-MS/MS (Agilent Technologies) approach was used to identify the chemical contents of SJC. Briefly, we weighed 1g SJC, and extracted the components with different gradients of organic solutions including ethanol, dichloromethane, $\mathrm{n}$-butyl alcohol, and ethyl acetate. All samples were dried and filtered before being separated on a Diamonsil C18 column $(250 \mathrm{~mm} \times 4.6 \mathrm{~mm}, 5 \mu \mathrm{m})$. The mobile phase was water containing $0.1 \%$ formic acid (A), and $100 \%$ acetonitrile (B) with the flow rate $1.0 \mathrm{~mL} / \mathrm{min}$. The mass spectrometry scan mode was at negative electrospray ionization mode (ESI-). The ESI parameters were set as follows: mass range, 100-1, $200 \mathrm{Da}$; scan time, $0.2 \mathrm{~s}$; gas flow rate, $6 \mathrm{~L} / \mathrm{min}$; cone voltage, $40 \mathrm{~V}$; capillary voltage, $3.5 \mathrm{k} \mathrm{V}$; collision voltage, $200 \mathrm{~V}$. The identification of compounds represented by each peak was carefully verified via positive controls, retention times, $m / z$ values, and fragments.

\section{Cell transfection and western blot}

GPR18-Tango plasmid was a gift from Dr. Bryan Roth's lab (Addgene plasmid \# 66340) and sub-cloned into pcDNA3.0 vector [14]. GPR18 specific siRNA was purchased from Santa Cruz Biotechnology (Cat. sc75171; Santa Cruz, CA) and transfected by using Lipofectamine 2000 according to the manufacturer's instructions [15]. Western blot assays were performed in order to evaluate the efficiency of GPR18 overexpression and the knockdown as described previously [16]. Mice peritoneal macrophages were homogenized in RIPA buffer to generate total cell lysates. After measuring the protein concentration via a bicinchoninic acid protein assay Kit, $20 \mu \mathrm{g}$ of extracted proteins was resolved on $10 \%$ sodium dodecyl sulfate polyacrylamide gel electrophoresis gels, transferred onto polyvinylidene fluoride membranes, blocked with 5\% non-fat milk, and probed with primary antibodies including GPR18 and $\beta$-actin (Abcam, Cambridge, MA). Horseradish peroxidase-conjugated secondary antibodies were then incubated for $1 \mathrm{~h}$ at room temperature followed by detection with ECL solution (Beyotime Biotechnology, Shanghai, China).

\section{cAMP assay}

GloSensor cells were transfected with siRNA or plasmids using Lipofectamine 2000 in 24-well dishes. Twenty-four hours after transfection, cells were plated on 96-well plates at a cell density of $2 \times 10^{4}$ cells/ well. Cells were maintained in culture medium for another $24 \mathrm{~h}$, then incubated with $100 \mu \mathrm{L}$ of medium containing 2\% (v/v) GloSensor cAMP reagent, $10 \% \mathrm{FBS}$, and $88 \% \mathrm{CO}_{2}$-independent medium for another $2 \mathrm{~h}$. The basal cAMP signal was measured using a luminescence counter. After the cAMP levels reached a steady baseline for $6 \mathrm{~min}, 1 \mu \mathrm{M}$ forskolin and verbenalin (or cannabinoid [CBD]) were added to determine the effects of forskolin-stimulated cAMP increase. For PTX treatment, $100 \mathrm{ng} / \mathrm{mL}$ PTX was preincubated with cells for at least $10 \mathrm{~h}$.

\section{Statistics}

Data are presented as the mean \pm standard deviation. Statistical analysis was performed using GraphPad Prism 5.0 Software (GraphPad Software Inc, CA), and statistical significance was evaluated using independent or paired Student's $t$-tests or one-way analysis of variance. A P value of less than 0.05 was considered statistically significant. 


\section{Cellular Physiology Cell Physiol Biochem 2018;50:629-639 \begin{tabular}{l|l|l} 
DOI: 10.1159/000494184 & $\begin{array}{l}\text { O 2018 The Author(s). Published by S. Karger AG, Basel } \\
\text { www.karger.com/cpb }\end{array}$ \\
\hline
\end{tabular}

Fig. 1. Effects of SJC on PAK infected mice lung tissues. Mice lung tissues were homogenized, and the expression levels of IL-6 (A) as well as IL-8 (B) were quantified the by ELISA method. The lung tissues from model mice group (which were infected with PAK but only treated with normal saline) showed a significant elevated IL-6 levels, while all the drug-treated groups exhibited lower IL-6 level compared with the model groups. Similarly, the IL-8 level was increased by PAK infection and can be attenuated by SJC. Mice in Lev group were intragastrically administered with levofloxacin $(75 \mathrm{mg} / \mathrm{kg}$ ); mice in SJC-L, SJC-M, SJC-H groups were intragastrically administered with SJC $(0.025 \mathrm{~g} / \mathrm{mL}, 0.05$ $\mathrm{g} / \mathrm{mL}$, and $0.1 \mathrm{~g} / \mathrm{mL}$, respectively); mice in control group and model group were treated with normal saline. All drug treatments were qd consistently for 2 weeks. Abbreviations: Con, control group; Mod, model group; Lev, levofloxacin; SJC-L, low Shufeng Jiedu Capsule concentration; SJC-M, middle Shufeng Jiedu Capsule concentration; SJC-H, high Shufeng Jiedu Capsule concentration.

\section{Results}

Histological inflammation in mice lung tissues were attenuated by SJC

We evaluated the pulmonary effects of SJC by measuring inflammatory factors such as IL- 6 and IL-8, which have been reported to be associated with acute lung infections $[17,18]$. Briefly, we homogenized the lung tissues and quantified the inflammatory factor levels by ELISA. The lung tissues from the Mod group showed significantly elevated IL-6 levels, while all drug-treated groups exhibited lower IL-6 levels compared with the Mod group (Fig. 1A). Similarly, IL-8 levels were increased after PAK infection and attenuated by SJC (Fig. 1B). The results indicated that SJC potentially inhibited PAK-induced acute lung infection (Table 1).

To further explore the possible mechanisms of PAK's function, we next used peritoneal macrophages from mice to test whether SJC could inhibit the secretion of inflammatory factors from macrophages. Lipopolysaccharide (LPS; $10 \mu \mathrm{g} / \mathrm{mL}$ ) was added during cell culture to induce the activation of macrophages. The LPS-treated cells showed significantly increased secretion of TNF- $\alpha$ (Fig. 2A). Notably, cells simultaneously treated with SJC secreted significantly lower amounts of TNF- $\alpha$. Another important inflammation factor, IL6 , was also monitored upon a different treatment and the results were consistent those for TNF- $\alpha$ (Fig. 2B, Table 2).

Gene sequencing of the mice lung tissues and SJC component identification

By comparing the microarray data among three groups (i.e., Con, Mod, and SJC-M groups), we identified 70 differentially expressed genes (Supplemental Fig. 1A, all $\log 2$ Ratio $>2$ or $<-2$ ). By loading the genes into String 9.1 software, we further determined the involvement of 84 pathways and 58 related genes (Supplemental Table 2). Among the pathways, up to 10 genes correlated with cytokine-receptor interplays, including Il21r, Ccl3, Cxcl10, Cxcr5, Pdgfra, Tnfrsf17, Tnfrsf13c, Tnf, Tnfrsf13b, and Il1r2 (Supplemental Fig. 1B). Additionally, the 
Pik3cg gene was closely associated with the migration of leukocytes. Interestingly, several GPCR family members, such as CXCR4 and GPR18, were also revealed to play central roles in the protein networks. From pathway analysis, we identified well-known inflammatory associated pathways including those of Toll-like receptor, B cell receptor, chemokines, and PPAR. Therefore, we generated possible anti-inflammatory networks for SJC (Supplemental Fig. 1C).

Before further exploring the altered genes and pathways from microarray experiments, we tried to precisely determine the chemical components in SJC by using HPLC-Q-TOFMS/MS. The mass spectrometry peaks are shown in Fig. 3. We initially detected up to 94 chemicals in the SJC powder (Supplemental Table 1). Among them, caffeic acid [19], epigoitrin [20], adenosine [21], uridine [22], guanosine [23], verbascoside [24], and isoliquiritigenin [25] have been shown to have anti-inflammatory effects by other groups. To elucidate the potential anti-inflammatory components, we used computer docking models to screen the compounds. The docking results showed that verbenalin, forsythoside A, phillyrin, vitexin, and emodin may possess potent anti-inflammatory powers.

\section{Verbenalin is a novel agonist of GPR18}

As described above, gene sequencing data identified several significant GPCRs, including GPR18. GPR18 is a cell surface receptor hypothetically linked to the ligand endocannabinoid $\mathrm{N}$-arachidonoyl glycine (NAGly) [26]. GPR18 is reported to be activated by a CBD agonist [27]. As one of the GPCR family members, the signaling activity of GPR18 was mediated by Gi protein, which subsequently inhibited adenylyl cyclase. We tested whether verbenalin could modulate inflammation via

GPR18 receptor. Through cAMP assays, we found that verbenalin exerts a similar stimulation curve with CBD (Fig. 4A), indicating its potential role as a ligand of GPR18. To further clarify the verbenalin-GPR18 signaling, we transfected GluSensor cells with GPR18-siRNA

Table 2. Secretion of cytokines in LPSinduced peritoneal macrophages

\begin{tabular}{lcc}
\hline Group & $\begin{array}{c}\text { TNF- } \alpha \text { level }(\mathrm{pg} / \mathrm{mL}) \\
\text { Mean } \pm \mathrm{SD}\end{array}$ & $\begin{array}{c}\mathrm{IL}-6 \text { level }(\mathrm{pg} / \mathrm{mL}) \\
\text { Mean } \pm \mathrm{SD}\end{array}$ \\
\hline Control & $29.51 \pm 15.08$ & $6.44 \pm 0.73$ \\
Model & $256.19 \pm 53.45$ & $495.91 \pm 33.89$ \\
Verbenalin-Low & $160.92 \pm 20.01$ & $483.06 \pm 14.80$ \\
Verbenalin-Middle & $97.59 \pm 26.07$ & $223.40 \pm 21.74$ \\
Verbenalin-High & $27.59 \pm 5.54$ & $106.54 \pm 12.11$ \\
\hline
\end{tabular}

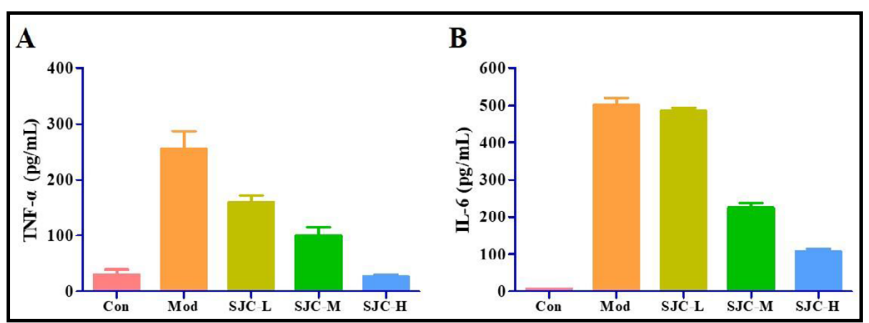

Fig. 2. SJC down-regulates secretion of TNF- $\alpha$ and IL-6 in mice peritoneal macrophages. The LPS-treated cells showed significant increased secretion of TNF- $\alpha$ (A) and IL-6 (B), while treatment with SJC attenuated the inflammation response. Cells in SJC-L, SJC-M, SJC-H groups were treated with SJC $0.1 \mathrm{mg} /$ $\mathrm{mL}, 1 \mathrm{mg} / \mathrm{mL}$, and $10 \mathrm{mg} / \mathrm{mL}$, respectively. Abbreviations: Con, control group; Mod, model group; Lev, levofloxacin; SJC-L, low Shufeng Jiedu Capsule concentration; SJC-M, middle Shufeng Jiedu Capsule concentration; SJC-H, high Shufeng Jiedu Capsule concentration.

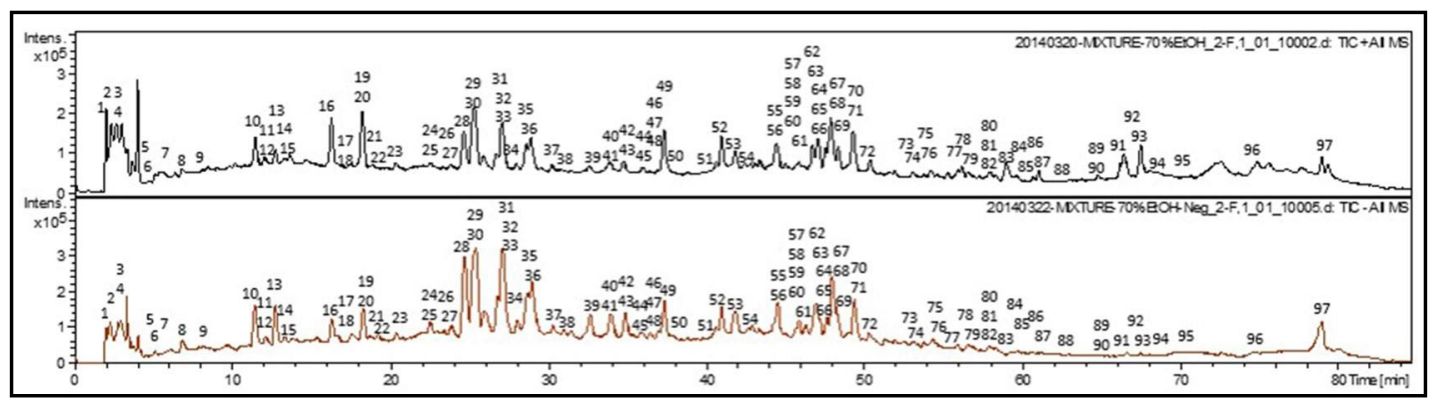

Fig. 3. Representative MS peaks from HPLC-TOF-MS/MS. Each Arabic number represents a peak with identified chemical compound (see also supplemental Table 1). 


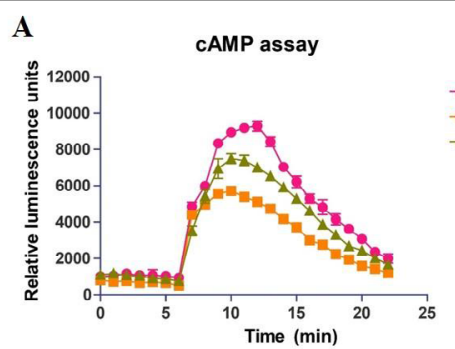

C

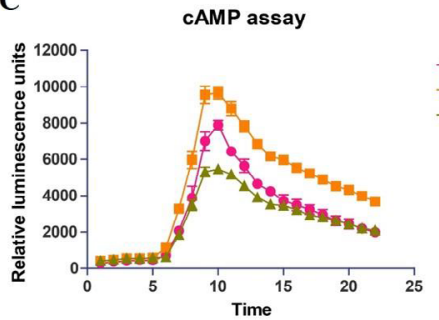

B

- Forskolin

- Forskolin+CBD

$\Perp$ Forskolin+Ver

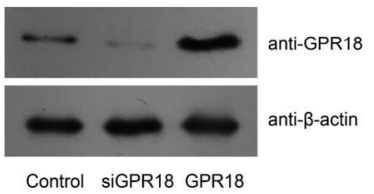

D

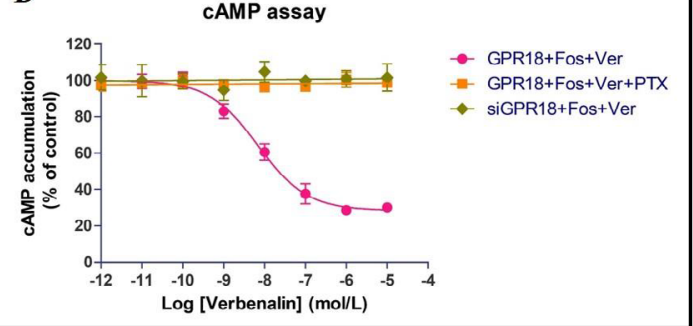

Fig. 4. Verbenalin can active GPR18-Gi signaling and inhibit cAMP production. (A) Comparison of cAMP production using GluSensor cells, both cannabidiol (CBD, $10 \mu \mathrm{M}$ ) and verbenalin (Ver, $10 \mu \mathrm{M})$ can attenuate the effect of forskolin (Fos, $1 \mu \mathrm{M}$ ) on activating adenylyl cyclase, indicating their potential role in Gi stimulation. (B) Transfection efficiency of GPR18 was tested by western blot. (C) Cells were simultaneously stimulated with forskolin and verbenalin. GPR18-silencing cells presented an increased cAMP accumulation compared with control cells. In contrast, GPR18-overexpression further enhance the effect of verbenalin. This data indicates that verbenalin may function through GPR18. (D) The dose-dependent effect of verbenalin on forskolin-stimulated cAMP accumulation was further tested. GPR18-overexpressed cells were stimulated with forskolin with $(\bullet)$ or without $(\bullet)$ pretreatment with PTX $(100 \mathrm{ng} / \mathrm{mL})$. Accumulation of cAMP was presented as a relative percentage of control in the absence of verbenalin. Cells with GPR18-siRNA $(\bullet)$ showed almost no effect on verbenalin stimulation.

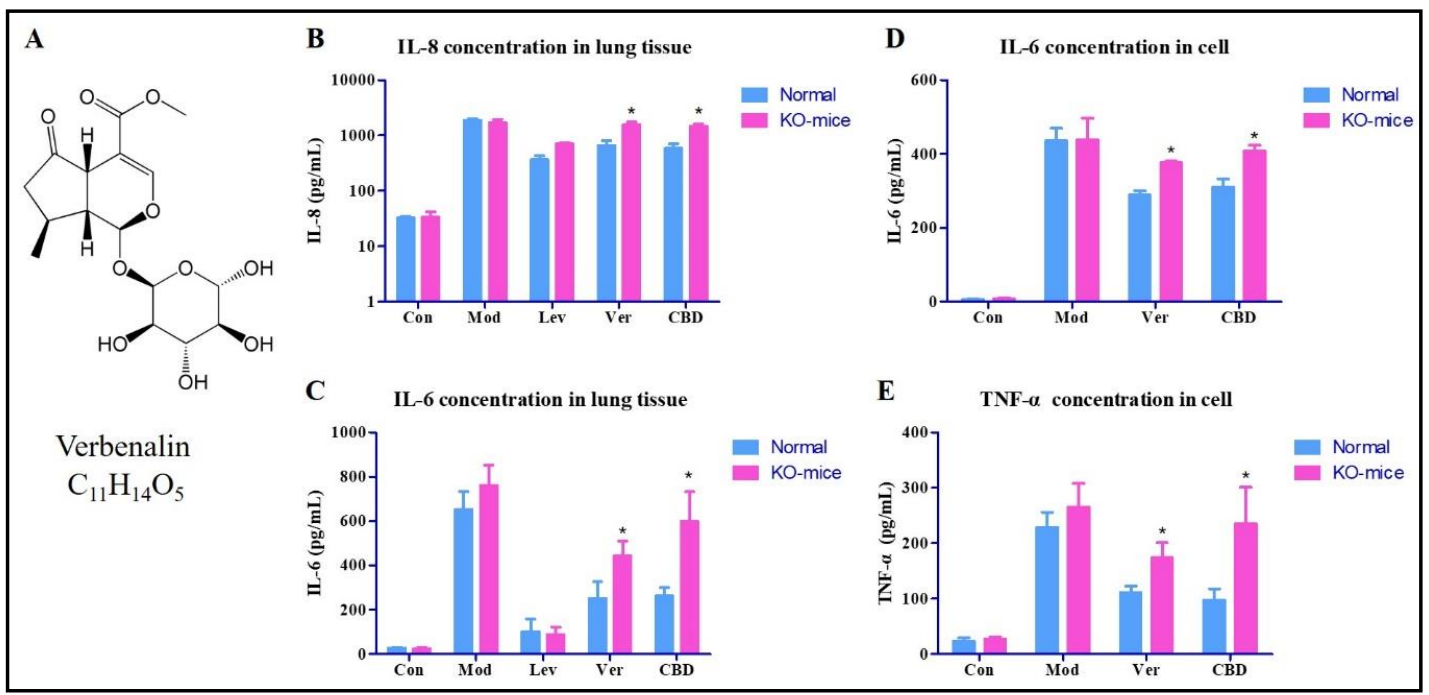

Fig. 5. Verbenalin is a potential anti-inflammatory compound in acute respiratory tract infection. (A) Chemical structure of verbenalin, which is classified as an iridoid glucoside. Verbenalin $(10 \mu \mathrm{M})$ showed significant effects on inhibiting the expression of IL-8 (B) and IL-6 (C) in PAK-induced mice lung tissues. However, its anti-inflammatory effects were attenuated in GPR18-KO mice compared with that of wild-type mice. Similarly, the secretion of IL-6 (D) and TNF- $\alpha$ (E) from isolated peritoneal macrophages were increased upon LPS-stimulation, and verbenalin treatment can decrease the levels of cytokines. Lev (Levofloxacin, 5 $\mu \mathrm{g} / \mathrm{mL}$ ), a non-GPR18 ligand, was used as negative control; CBD (Cannabidiol, $10 \mu \mathrm{M}$ ), a known agonist for GPR18, was used as positive control. 


\section{Cellular Physiology Cell Physiol Biochem 2018;50:629-639 \begin{tabular}{ll|l} 
and Biochemistry Published online: 12 October 2018 & $\begin{array}{l}\text { (c) } 2018 \text { The Author(s). Published by S. Karger AG, Basel } \\
\text { www.karger.com/cpb }\end{array}$
\end{tabular}

(siGPR18) or GPR18-plasmids (GPR18 overexpression) (Fig. 4B). Interestingly, cells that overexpressed GPR18 showed more significant inhibitory effects on cAMP signaling with verbenalin, while cells with siGPR18 showed attenuated Gicoupled signaling (Fig. 4C). In addition, we used PTX, a Gi protein inhibitor to further elucidate the signaling pathway, which showed a complete block on the effects of verbenalin in GPR18-overexpression cells (Fig. 4D). Therefore, we came to the primary conclusion that verbenalin could directly activate GPR18.

\section{Verbenalin attenuates inflammation by} targeting GPR18

We next wanted to test the independent effects of verbenalin on the PAK-infected mouse model and found it effectively inhibited the expression of IL-8 and IL-6 (Fig. 5A-C). In addition to verbenalin, we also confirmed the anti-inflammatory role of GPR18. Our data found no statistically significant differences in IL-8 or IL-6 expression levels in PKA infected lung tissues between WT and GPR18-KO mice. Nevertheless, the results indicated that verbenalin had different effects on the two types of mice (Fig. 5A-C). More specifically, verbenalin exhibited better anti-inflammatory effects in WT mice than in GPR18-KO mice (Table 3). The effect of CBD was tested as a positive control.

We isolated peritoneal macrophages from both WT and GPR18-KO mice, and stimiated them with LPS. The inhibitory effect of verbenalin or CBD on the secretion of inflammatory factors in macrophages was attenuated in cells derived from GPR-18 KO mice (Fig. 5D, 5E, Table 4).

\section{Discussion}

SJC comprises Polygonum cuspidatum, Fructus forsythia, Radix isatidis, Radix bupleuri, Dahurian patrinia herb, Verbena, Rhizoma phragmitis and Liquorice root. SJC is widely used as an antipyretic and anti-inflammatory medicine [6,28-30], especially for the treatment of acute upper respiratory tract infections [31], which may involve the ERK signaling pathway. Although it has shown significant effects for the treatment of sore throat, headache, nasal congestion, flow turbidity, and pulmonary embolism, most other studies on SJC have focused on single chemical composition analysis. The systematic components, as well as the detailed effective mechanisms of SJC, have not been clearly reported. Thus, in this study, we confirmed that 94 compounds were present in SJC via MS. These included 7 amino acids, 7 saccharides, 7 olefinic esters, 11 phenylethylglycosides, 1 stilbene, 24 flavonoids, 6 lignans, 6 anthraquinones, 18 triterpenoids, 1 coumarin, 5 alkaloids, and 2 other small molecules. Our data provided new information on the material basis of drug function in order to improve drug efficiency.

Among the identified compounds, verbenalin was reported to be effective on a prostatitis mouse model [32]. The group tested the number of white cells and the lecithin corpuscle density in prostatic fluid by giving high, medium, and low doses of verbenalin. According to morphological observations, they demonstrated that verbenalin can significantly reduce pathological prostatitis changes compared with a control group. Since verbenalin has
Table 3. Expression levels of cytokines in lung

Table 4. Expression levels of cytokines in peritoneal macrophages

\begin{tabular}{lcccc}
\hline Group & \multicolumn{2}{c}{$\begin{array}{c}\text { TNF- } \alpha \text { level }(\mathrm{pg} / \mathrm{mL}) \\
\text { Mean } \pm \text { SD }\end{array}$} & \multicolumn{2}{c}{$\begin{array}{c}\text { IL-6 level }(\mathrm{pg} / \mathrm{mL}) \\
\text { Mean } \pm \text { SD }\end{array}$} \\
& $\begin{array}{c}\text { Wild type } \\
\text { mice }\end{array}$ & GPR18-KO mice & $\begin{array}{c}\text { Wild type } \\
\text { mice }\end{array}$ & GPR18-KO mice \\
\hline Control & $23.36 \pm 8.68$ & $27.15 \pm 6.24$ & $6.47 \pm 1.09$ & $8.31 \pm 2.74$ \\
Model & $228.47 \pm 39.21$ & $265.08 \pm 60.21$ & $435.58 \pm 49.16$ & $438.42 \pm 81.82$ \\
Verbenalin & $111.16 \pm 18.59$ & $173.71 \pm 38.43$ & $289.03 \pm 15.68$ & $376.41 \pm 5.85$ \\
Cannabidiol & $89.38 \pm 38.66$ & $208.71 \pm 38.43$ & $314.22 \pm 26.52$ & $412.89 \pm 22.34$ \\
\hline
\end{tabular}




\section{Cellular Physiology Cell Physiol Biochem 2018;50:629-639 \begin{tabular}{ll|l} 
DOI: 10.1159/000494184 & $\begin{array}{l}\text { O } 2018 \text { The Author(s). Published by S. Karger AG, Basel } \\
\text { www.karger.com/cpb }\end{array}$
\end{tabular} \\ Yuan et al.: Verbenalin Inhibits Lung Inflammation by GPR18}

shown good therapeutic effects in the prostatitis mouse model, we were curious to further investigate whether it functions to inhibit acute upper respiratory tract infection. We first confirmed the role of SJC for its ability to down-regulate the expression of IL-8 and IL-6 in lung tissues due to PAK-infection in mice. IL-8 and IL- 6 are well-known cytokines that can cause fever and inflammation. Interestingly, treatment with pure verbenalin compound also showed significant inhibitory effects on the expression of TNF- $\alpha$ and IL-6, revealing its novel role in the regulation of upper respiratory tract infections. Similarly, cellular experiments using peritoneal macrophages further confirmed our conclusion.

Furthermore, by using CBD as a positive control, our data originally identified verbenalin as a novel ligand. Through the use of GPR18 and taking into consideration that GPR18 and its ligand $\mathrm{N}$-arachidonoylglycine are potential regulators of inflammation [33,34], we next explored whether GPR18 plays a role in acute upper respiratory tract infections. After we infected the model mice with PAK, we did not find any differences in basal inflammatory factor expression in lung tissues between WT and GPR18-KO mice. On the other hand, verbenalin showed inhibitory effects on inflammation responses in GPR18-KO mice, but this effect was significantly weaker than that in WT mice, indicating the role of GPR18 in the antiinflammatory effects or verbenalin. Our results are consistent with a previous study, which showed that GPR18 regulates the reconstitution of mouse small intestine intraepithelial lymphocytes [35]. Other reported functions of GPR18 include its modulation of myocardial function, blood pressure, and intraocular pressure [36, 37]. Therefore, our results also suggested further possible applications of verbenalin in the treatment of related diseases. More in-depth studies are necessary to verify the roles and mechanisms of verbenalinGPR18 signaling pathway.

Besides verbenalin, our study revealed other compounds with anti-inflammatory activity, such as caffeic acid, epigoitrin, and guanosine in SJC. Further studies will be required to investigate their specific roles and combined effects in the treatment of acute respiratory tract infections.

\section{Conclusion}

Our data not only determined the chemical composition of SJC, but also showed that verbenalin is a significant anti-inflammatory compound and may function through GPR18 membrane receptor.

\section{Disclosure Statement}

All authors declare they have no conflicts of interest.

\section{References}

1 Li Y, Huang J, Li L, Liu L: Synergistic activity of berberine with Azithromycin against Pseudomonas aeruginosa isolated from patients with cystic fibrosis of lung in vitro and in vivo. Cell Physiol Biochem 2017;42:1657-1669.

-2 Riethmüller J, Herrmann G, Graepler-Mainka U, Hellwig D, Heuer H-E, Heyder S, Köster H, Kinder B, Kröger K, Paul K: Sequential inhalational tobramycin-colistin-combination in CF-patients with chronic P. aeruginosa colonization-an observational study. Cell Physiol Biochem 2016;39:1141-1151.

-3 Tabazavareh ST, Seitz A, Jernigan P, Sehl C, Keitsch S, Lang S, Kahl BC, Edwards M, Grassmé H, Gulbins E: Lack of sphingosine causes susceptibility to pulmonary Staphylococcus aureus infections in cystic fibrosis. Cell Physiol Biochem 2016;38:2094-2102. 


\section{Cellular Physiology Cell Physiol Biochem 2018;50:629-639 \begin{tabular}{ll|l} 
and Biochemistry Published online: 12 October 2018 & $\begin{array}{l}\text { (c) } 2018 \text { The Author(s). Published by S. Karger AG, Basel } \\
\text { www.karger.com/cpb }\end{array}$
\end{tabular}}

Yuan et al.: Verbenalin Inhibits Lung Inflammation by GPR18

4 Shapiro DJ, Hicks LA, Pavia AT, Hersh AL: Antibiotic prescribing for adults in ambulatory care in the USA, 2007-09. J Antimicrob Chemother 2014;69:234-240.

5 Becker KA, Li X, Seitz A, Steinmann J, Koch A, Schuchman E, Kamler M, Edwards MJ, Caldwell CC, Gulbins E: Neutrophils Kill Reactive Oxygen Species-Resistant Pseudomonas aeruginosa by Sphingosine. Cell Physiol Biochem 2017;43:1603-1616.

6 Xi Z, Zhou J, Mei J, Tan S, Ge A, Zheng Y, Chen F, Zhao Q Geng L, Shao M: Effect of Shufeng Jiedu Capsule on the fever caused by viral upper respiratory tract infection: a report of 130 cases. J Tradit Chin Med 2010;51:426-427.

-7 Bao Y, Gao Y, Cui X: Effect of Shufeng Jiedu capsules as a broad-spectrum antibacterial. Biosci Trends 2016;10:74-78.

-8 Tao Z, Meng X, Han YQ, Xue MM, Wu S, Wu P, Yuan Y, Zhu Q, Zhang TJ, Wong CC: Therapeutic mechanistic studies of ShuFengJieDu capsule in an acute lung injury animal model using quantitative proteomics technology. J Proteome Res 2017;16:4009-4019.

-9 Ramphal R, Balloy V, Jyot J, Verma A, Si-Tahar M, Chignard M: Control of Pseudomonas aeruginosa in the lung requires the recognition of either lipopolysaccharide or flagellin. J Immunol 2008;181:586-592.

10 Balloy V, Verma A, Kuravi S, Si-Tahar M, Chignard M, Ramphal R: The role of flagellin versus motility in acute lung disease caused by Pseudomonas aeruginosa. J Infect Dis 2007;196:289-296.

11 Bao Y, Gao Y, Cui X: Effect of Shufeng Jiedu capsules as a broad-spectrum antibacterial. Biosci Trends 2016;10:74-78.

12 Kim MJ, Nepal S, Lee ES, Jeong TC, Kim SH, Park PH: Ethanol increases matrix metalloproteinase-12 expression via NADPH oxidase-dependent ROS production in macrophages. Toxicol Appl Pharmacol 2013;273:77-89.

13 Yang Y, Dong R, Hu D, Chen Z, Fu M, Wang DW, Xu X, Tu L: Liver Kinase B1/AMP-Activated Protein Kinase Pathway Activation Attenuated the Progression of Endotoxemia in the Diabetic Mice. Cell Physiol Biochem 2017;42:761-779.

14 Kroeze WK, Sassano MF, Huang XP, Lansu K, McCorvy JD, Giguere PM, Sciaky N, Roth BL: PRESTO-Tango as an open-source resource for interrogation of the druggable human GPCRome. Nat Struct Mol Biol 2015;22:362-369.

15 Tan W, Pan M, Liu H, Tian H, Ye Q Liu H: ergosterol peroxide inhibits ovarian cancer cell growth through multiple pathways. Onco Targets Ther 2017;10:3467.

16 Liu H, Xu Y, Zhang Q, Li K, Wang D, Li S, Ning S, Yang H, Shi W, Liu Z: Correlations between TBL1XR1 and recurrence of colorectal cancer. Sci Rep 2017;7:44275.

17 Xing Z, Gauldie J, Cox G, Baumann H, Jordana M, Lei XF, Achong MK: IL-6 is an antiinflammatory cytokine required for controlling local or systemic acute inflammatory responses. J Clin Invest 1998;101:311.

18 Ordonez CL, Shaughnessy TE, Matthay MA, Fahy JV: Increased neutrophil numbers and IL-8 levels in airway secretions in acute severe asthma: clinical and biologic significance. Am J Respir Crit Care Med 2000;161:1185-1190.

19 Michaluart P, Masferrer JL, Carothers AM, Subbaramaiah K, Zweifel BS, Koboldt C, Mestre JR, Grunberger D, Sacks PG, Tanabe T: Inhibitory effects of caffeic acid phenethyl ester on the activity and expression of cyclooxygenase- 2 in human oral epithelial cells and in a rat model of inflammation. Cancer Res 1999;59:2347-2352.

20 Shin EK, Kim DH, Lim H, Shin H-K, Kim J-K: The anti-inflammatory effects of a methanolic extract from Radix Isatidis in murine macrophages and mice. Inflammation 2010;33:110-118.

21 Reutershan J, Vollmer I, Stark S, Wagner R, Ngamsri K-C, Eltzschig HK: Adenosine and inflammation: CD39 and CD73 are critical mediators in LPS-induced PMN trafficking into the lungs. FASEB J 2009;23:473-482.

22 Evaldsson C, Ryden I, Uppugunduri S: Anti-inflammatory effects of exogenous uridine in an animal model of lung inflammation. Int Immunopharmacol 2007;7:1025-1032.

23 Jiang S, Bendjelloul F, Ballerini P, D’Alimonte I, Nargi E, Jiang C, Huang X, Rathbone MP: Guanosine reduces apoptosis and inflammation associated with restoration of function in rats with acute spinal cord injury. Purinergic Signal 2007;3:411-421.

24 Esposito E, Mazzon E, Paterniti I, Dal Toso R, Pressi G, Caminiti R, Cuzzocrea S: PPAR-contributes to the anti-inflammatory activity of verbascoside in a model of inflammatory bowel disease in mice. PPAR Res 2010;2010:917312. 


\section{Cellular Physiology Cell Physiol Biochem 2018;50:629-639

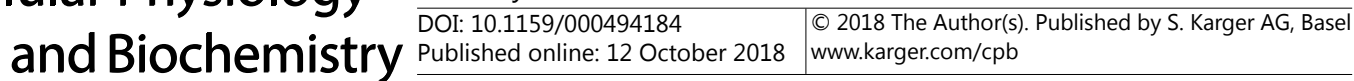 \\ Yuan et al.: Verbenalin Inhibits Lung Inflammation by GPR18}

-25 Honda H, Nagai Y, Matsunaga T, Okamoto N, Watanabe Y, Tsuneyama K, Hayashi H, Fujii I, Ikutani M, Hirai Y: Isoliquiritigenin is a potent inhibitor of NLRP3 inflammasome activation and diet-induced adipose tissue inflammation. J Leukoc Biol 2014;96:1087-1100.

26 Kohno M, Hasegawa H, Inoue A, Muraoka M, Miyazaki T, Oka K, Yasukawa M: Identification of $\mathrm{N}$-arachidonylglycine as the endogenous ligand for orphan G-protein-coupled receptor GPR18. Biochem Biophys Res Commun 2006;347:827-832.

27 Console-Bram L, Brailoiu E, Brailoiu GC, Sharir H, Abood ME: Activation of GPR18 by cannabinoid compounds: a tale of biased agonism. Br J Pharmacol 2014;171:3908-3917.

-28 Tao Z, Gao J, Zhang G, Xue M, Yang W, Tong C, Yuan Y: Shufeng Jiedu Capsule protect against acute lung injury by suppressing the MAPK/NF-кB pathway. Biosci Trends 2014;8:45-51.

29 Ying Y, Qingwu L, Mingming X, Zhenju S, Chaoyang T, Zhengang T: Emodin: One Main Ingredient of Shufeng Jiedu Capsule Reverses Chemoresistance of Lung Cancer Cells Through Inhibition of EMT. Cell Physiol Biochem 2017;42:1063-1072.

30 Kovacic B, Sehl C, Wilker B, Kamler M, Gulbins E, Becker KA: Glucosylceramide critically contributes to the host defense of cystic fibrosis lungs. Cell Physiol Biochem 2017;41:1208-1218.

31 Li Y, Chang N, Han Y, Zhou M, Gao J, Hou Y, Jiang M, Zhang T, Bai G: Anti-inflammatory effects of Shufengjiedu capsule for upper respiratory infection via the ERK pathway. Biomed Pharmacother 2017;94:758-766.

-32 Miao M, Guo L, Yan X, Wang T, Li Z: Effects of verbenalin on prostatitis mouse model. Saudi J Biol Sci 2016;23:S148-157.

33 Takenouchi R, Inoue K, Kambe Y, Miyata A: N-arachidonoyl glycine induces macrophage apoptosis via GPR18. Biochem Biophys Res Commun 2012;418:366-371.

-34 Burstein SH, McQuain CA, Ross AH, Salmonsen RA, Zurier RE: Resolution of inflammation by N-arachidonoylglycine. J Cell Biochem 2011;112:3227-3233.

-35 Becker AM, Callahan DJ, Richner JM, Choi J, DiPersio JF, Diamond MS, Bhattacharya D: GPR18 Controls Reconstitution of Mouse Small Intestine Intraepithelial Lymphocytes following Bone Marrow Transplantation. PLoS One 2015;10:e0133854.

-36 Matouk AI, Taye A, El-Moselhy MA, Heeba GH, Abdel-Rahman AA: The Effect of Chronic Activation of the Novel Endocannabinoid Receptor GPR18 on Myocardial Function and Blood Pressure in Conscious Rats. J Cardiovasc Pharmacol 2017;69:23-33.

-37 Miller S, Leishman E, Oehler O, Daily L, Murataeva N, Wager-Miller J, Bradshaw H, Straiker A: Evidence for a GPR18 Role in Diurnal Regulation of Intraocular Pressure. Invest Ophthalmol Vis Sci 2016;57:6419-6426. 